\title{
TO STUDY SEVERITY AND RISK PREDICTION OF MORTALITY IN MALARIA BY THE MALARIA SEVERITY SCORE
}

Aharwar $\mathrm{S}^{1}$, Kansal A2 ${ }^{2}$ Jatav $0 . \mathrm{P}^{3}$, Trikha $\mathrm{S}^{4}$, Malviya $\mathrm{K}^{5}$

\section{HOW TO CITE THIS ARTICLE:}

Aharwar S, Kansal A, Jatav O. P, Trikha S, Malviya K. "To Study Severity and Risk Prediction of Mortality in Malaria by the Malaria Severity Score". Journal of Evolution of Medical and Dental Sciences 2014; Vol. 3, Issue 07, February 17; Page: 1796-1810, DOI: $10.14260 /$ jemds/2014/2060

ABSTRACT: AIMS AND OBJECTIVES: Malaria still remains today as it has been for centuries, one of the most serious parasitic diseases of the world affecting 300-500 million people and causing over 1 million deaths each year. Research on objective assessment of disease severity and prediction of mortality risk in malaria is lacking even if it frequently develops multiple organ dysfunction (MOD) during the course of illness. Therefore the aim of the present study is to apply a severity score for assessment of disease severity and risk prediction in adult patients of severe malaria. MATERIAL AND METHODS: This open prospective study was conducted in the department of medicine, G.R. Medical College \& J.A. Group of Hospital, Gwalior (M.P.), India comprising of 130 cases of malaria who was admitted in the Department from June 2010 to Oct. 2011. All the patients were evaluated for the various clinical presentations as per a predefined proforma. All the patients will investigated for CBC, S. Bilirubin, SGOT, SGPT, urea, creatinine, Serum proteins, S. electrolytes and then put on the Malaria severity score of Mohapatra et al for the assessment of Prognosis and mortality. RESULTS AND DISCUSSION: In present study according of Mohapatra severity score we assessed organ dysfunction from the variables obtained from malaria patients. We obtained 31 cases without any organ dysfunction without any mortality and 36 cases of 1 organ dysfunction without any mortality. There were 23 cases of 2 organ dysfunction with 1 mortality (4.3\%) with score 10.18 cases of 3 organ dysfunction with $1(5.5 \%)$ mortality with score 11.8 cases of the 4 organ dysfunction with 2 mortality (25\%) with score 12 and 13.5 cases of the 5 organ dysfunction with 3 mortality (60\%) 1 mortality with malaria severity score 15 and other 2 mortality with malaria severity score $14 \& 16$ respectively. 5 cases of the 6 organ dysfunction with 4 mortality (80\%) 1 mortality with score 16 and second mortality with score 17 and other 2 mortality with score 18.4 cases of the 7 organ dysfunction with 4 mortality (100\%) 1 mortality with 19 score and second mortality with malaria severity score 20 and other two mortality with score 21 with $p$ value $0.0001, \chi^{2}=41.5$. CONCLUSION: Incidence of mortality significantly increases with increase in the malaria severity score with $p$ value 0.0001 . Increase probability of mortality in malaria patient is associated increase number of organ involvement with increase in the severity score. Assessment of malaria severity score help in assessing severity of organ dysfunction and it helps in risk stratification, prognosis and planning of the treatment to arrest the progression of disease as it is cheap \& effective it should be done in all malaria patients.

KEYWORDS: Plasmodium vivax, Plasmodium falciparum, multiple organ dysfunction.

INTRODUCTION: Malaria still remains today as it has been for centuries, one of the most serious parasitic diseases of the world affecting 300-500 million people and causing over 1 million deaths each year. ${ }^{1}$ Malaria is a major cause of mortality and morbidity in the tropical and subtropical regions of the world. An estimated 3.3 billion people were living in areas at risk of malaria in 2006. In India, 
the total no. of malaria cases in 2006 was 1.67 million. Out of which 0.75 million were due to P. falciparum malaria. Total 1487 deaths were reported due to malaria in $2006 .{ }^{2}$

Of the five species of plasmodia causing human malaria, P. falciparum causes the most serious form of the diseases. Infections with this parasite may become severe and fatal without early diagnosis and prompt and appropriate case management. Prompt action is especially important for high risk groups such as young children and pregnant women.

The P. falciparum infection can lead to cerebral malaria, acute renal failure, acute malarial hepatitis, hypoglycemia, hyperpyrexia, non-cardiogenic pulmonary edema, adult respiratory distress syndrome, adrenal insufficiency-like syndrome, hyperparasitemia, Blackwater fever, cardiac arrhythmias and gastrointestinal syndromes like secretory diarrhea ${ }^{3-4}$. Severe P. vivax malaria manifestations that have been reported are cerebral malaria, severe anemia, severe thrombocytopenia and pancytopenia, jaundice, splenic rupture, acute renal failure and acute respiratory distress syndrome. Severe anemia and acute pulmonary edema are not uncommon. ${ }^{2}$

Research on objective assessment of disease severity and prediction of mortality risk in malaria is lacking even though it frequently develops multiple organ dysfunction (MOD) during the course of illness. There is also paucity of standardized criteria to define organ dysfunction and to estimate risk for mortality objectively in patients of malaria ${ }^{5,6}$. Objective risk assessment is very useful for clinical decision making, in evaluating new therapies, in improving quality of treatment.

In such a situation assessment of severity of organ dysfunction is required for risk stratification, prognostication and planning of treatment to arrest the progression of disease.

Therefore the aim of the present study is to apply a severity score ${ }^{7}$ of Mohapatra et al for assessment of disease severity and risk prediction in adult patients of severe malaria.

MATERIAL AND METHODS: This open prospective study was conducted in the department of medicine, G.R. Medical College \& J.A. Group of Hospital, Gwalior (M.P.), India on the patients admitted from June 2010 to Oct. 2011. Adult patients with age >18yrs presenting with fever, headache, body ache confirmed malaria by peripheral blood smear and/or by fluorescence antigen antibody detection method. Known case of co- morbid disorder like chronic liver disorder, Diabetes Mellitus, chronic renal failure, rheumatic heart disease, coronary artery disease, sickle cell disease and associated infections like pneumonia, urinary tract infection and viral hepatitis and pregnancy were excluded from the study. After exclusion 130 case of malaria were selected for the study.

Clinical findings, hematological and biochemical investigations were entered in pre-designed proforma and were put on malaria severity score designed by Mohapatra et $\mathrm{al}^{7}$ as below and score was calculated and prognosis was assessed. We have taken malaria severity score of Mohapatra et al as they have developed score on the basis of large cohort of patients as compared to other studies ${ }^{8}$. Though many organ systems may be affected by falciparum malaria, from previous studies it was found that 7 major OD (table A) occurred commonly in malaria. ${ }^{4}$ Therefore, in the present study 7 OD with different levels of severity had been defined and validated. Organ dysfunction in malaria can be defined quantitatively by taking different variables into consideration. The use of single or multiple variables for definition of OD has been based on statistical methods that analyzed the ranges and relative weights of different variables for defining a particular OD which is similar to sepsis9,10. Each OD has been assigned a gradation of severity and allocated a score. Hence, the severity of each OD can be determined quantitatively with the corresponding score and the total score can be obtained by 
adding the scores of individual OD present in the patient, subsequently that can be converted to a risk estimate of mortality. For assessment of severity 12 different variables (table B) were studied. The variables were analyzed for defining organ dysfunction (OD) and assessment of the severity of complications. Patients were examined and assessed twice daily until full recovery or death.

In malaria severity score of Mohapatra, all 12 variables were analyzed to identify cut off points that define normal and abnormal values. Further from the abnormal values, different level of severity for each variable was determined. When the values were within the normal range it was considered as level-0 severity. 3 levels of increasing severity i.e. Level-I, II, and III were identified. Variable related to one system were grouped together to define that particular system.

A severity score of $0,1,3$, and 5 was assigned to 0 , I, II, and III level of severity respectively (table C). The score may be as low as 1 or as high as 5 for neurologic and renal involvement. For cardiovascular, respiratory, and hematological system the lowest and the highest score was 1 and 3. For hepatic and metabolic involvement only 1 score was assigned.

Organ dysfunction with only one level (I), two levels (I and II), and three levels (I to III) of severity were considered as least, moderate, and most severe dysfunction.

The points for each organ dysfunction are summed to calculate the total malaria severity score (MSS). MSS of 0 indicates no organ dysfunction, whereas MSS of 1 is the lowest level of severity possible for one single organ dysfunction. The maximum score with 21 points can be found when a patient has all 7 organs dysfunction with their highest level of severity.

\begin{tabular}{|c|c|c|}
\hline Criteria & \multicolumn{2}{|l|}{ Parameters } \\
\hline (a) General & \multicolumn{2}{|l|}{$\begin{array}{l}\text { 1. Fever }>101^{\circ} \mathrm{F} \\
\text { 2. Presence of malaria parasite }\end{array}$} \\
\hline \multirow[t]{8}{*}{ (b) Organ Specific } & Organ System & Parameters for defining dysfunction \\
\hline & 1. Neurologic & (a) Glasgow Coma scale $<13$ \\
\hline & 2. Renal (one or more) & $\begin{array}{l}\text { (a) Urine output }<750 \mathrm{ml} / 24 \mathrm{hr} \text {. } \\
\text { (b) S-creatinine }>1.2 \mathrm{mg} / \mathrm{dl} \\
\text { (c) } \mathrm{B} . \text { Urea }>36.0 \mathrm{mg} / \mathrm{dl}\end{array}$ \\
\hline & 3. Hepatic & Serum Bilirubin $>2$ \\
\hline & 4. Respiratory & (a) Respiratory rate $>30 /$ minute \\
\hline & 5. Cardiac (one or more) & $\begin{array}{l}\text { (a) Systolic blood pressure } \leq 90 \mathrm{~mm} \mathrm{Hg} \text {. } \\
\text { (b) Heart rate } \geq 120 \text { beats / minute or }<51 \text {. }\end{array}$ \\
\hline & 6. Metabolic & (a) B. Glucose $\leq 60 \mathrm{mg} / \mathrm{dL}$ \\
\hline & $\begin{array}{l}\text { 7. Hematological (one or } \\
\text { more) }\end{array}$ & $\begin{array}{l}\text { (a) Hemoglobin }<10.0 \mathrm{gm} . / \mathrm{dL} \\
\text { (b) Platelet count }<80,000 / \mu \mathrm{L} \\
\text { (c) Total leucocyte count }<4000 / \mu \mathrm{L} \text { or }> \\
12,000\end{array}$ \\
\hline
\end{tabular}


ORIGINAL ARTICLE

\begin{tabular}{|c|c|c|c|c|c|}
\hline \multirow{2}{*}{\multicolumn{2}{|c|}{$\begin{array}{c}\text { Parameters of } \\
\text { Organ Dysfunction }\end{array}$}} & \multicolumn{4}{|c|}{ Range of variables for different Level of severity } \\
\hline & & \multirow{2}{*}{\begin{tabular}{|c|} 
Level -0 \\
$14-15$
\end{tabular}} & \multirow{2}{*}{$\begin{array}{l}\text { Level I } \\
10-13\end{array}$} & \multirow{2}{*}{$\begin{array}{c}\text { Level - II } \\
7-9\end{array}$} & \multirow{2}{*}{$\begin{array}{c}\text { Level - III } \\
0-6\end{array}$} \\
\hline 1 & Neurologic GCS score & & & & \\
\hline 2 & $\begin{array}{l}\text { Renal } \\
\text { B. Urea (mg/dl) } \\
\text { S. Creatinine (mg/dl) } \\
\text { Urine Output (L/day) }\end{array}$ & $\begin{array}{c}10.0-36.0 \\
0.6-1.2 \\
0.75-3.9\end{array}$ & $\begin{array}{c}37.0-59.0 \\
1.3-1.9 \\
0.5-0.75\end{array}$ & $\begin{array}{c}60.0-119.0 \\
2.0-4.9 \\
0.4-0.5\end{array}$ & $\begin{array}{c}>120.0 \\
>5.0 \\
<0.5\end{array}$ \\
\hline 3 & $\begin{array}{l}\text { Cardiovascular } \\
\text { Heart rate/min } \\
\text { Systolic Blood Pressure } \\
\text { (mmHg) }\end{array}$ & $\begin{array}{l}51-119 \\
90-160\end{array}$ & $\begin{array}{c}120-139 \\
70-89\end{array}$ & $\begin{array}{c}>140 \text { or }<51 \\
41-69\end{array}$ & \\
\hline 4 & $\begin{array}{l}\text { Respiratory } \\
\text { Respiration rate/min }\end{array}$ & $20-30$ & $31-40$ & $>41$ & \\
\hline 5 & $\begin{array}{l}\text { Hematologic } \\
\mathrm{Hb} .(\mathrm{g} / \mathrm{dl}) \\
\mathrm{TLC}(/ \mathrm{cmm}) \\
\text { Platelet }(/ \mathrm{cmm})\end{array}$ & $\begin{array}{c}10.0-13.9 \\
4001-16,000 \\
80,000-2,50,000\end{array}$ & $\begin{array}{c}7.0-9.9 \\
2001-4000 \text { or } \\
10-20000 \\
<80,000\end{array}$ & $\begin{array}{l}<7.0 \\
<2000\end{array}$ & \\
\hline 6 & $\begin{array}{l}\text { Hepatic } \\
\text { S. Bilirubin (mg/dl) }\end{array}$ & $<2.0$ & $>2.0$ & & \\
\hline 7 & $\begin{array}{l}\text { Metabolic } \\
\text { B. Glucose (mg/dl) } \\
\mathrm{Na}+ \\
\mathrm{K}+\end{array}$ & $\begin{array}{l}60.0-110.0 \\
\quad<135 \\
<3.5 \text { to } 5.5\end{array}$ & $\begin{array}{c}<60.0 \\
<130 \\
<3\end{array}$ & & \\
\hline
\end{tabular}

\begin{tabular}{|l|c|c|c|c|}
\hline \multirow{2}{*}{\begin{tabular}{c} 
Organ Dysfunction \\
\multicolumn{1}{c|}{ and Score }
\end{tabular}} & \multicolumn{4}{c|}{ Level of severity } \\
\cline { 2 - 5 } & $\mathbf{0}$ & I & II & III \\
\hline Neurologic & Score-0 & Score-1 & Score-3 & Score-5 \\
\hline Renal & Score-0 & Score-1 & Score-3 & Score-5 \\
\hline Cardiovascular & Score-0 & Score-1 & Score-3 & \\
\hline Respiratory & Score-0 & Score-1 & Score-3 & \\
\hline Hematologic & Score-0 & Score-1 & Score-3 & \\
\hline Hepatic & Score-0 & Score-1 & & \\
\hline Metabolic & Score-0 & Score-1 & & \\
\hline
\end{tabular}

Table C: M.S.S. of each organ dysfunction with different level of severity

OBSERVATION: Out of 130 cases 70(53.84\%) patient were having plasmodium falciparum \& $58(44.61 \%)$ were having plasmodium vivax and $2(1.53 \%)$ patients have mixed (table 1 ). 


\begin{tabular}{|c|c|c|}
\hline Type of malaria & Number of Patients & \% CASE \\
\hline P.Falciparum & 70 & $53.84 \%$ \\
\hline P.vivax & 58 & $44.61 \%$ \\
\hline Mixed & 2 & $1.53 \%$ \\
\hline Total & 130 & $100 \%$ \\
\hline
\end{tabular}

Of these $81(62.30 \%)$ were male and $49(37.69 \%)$ were female (table 2$)$.

\begin{tabular}{|l|c|c|c|}
\hline \multicolumn{1}{|c|}{ Total cases } & Male & Female & Total \\
\hline P.Falciparum & $41(31.53 \%)$ & $29(22.30 \%)$ & $70(53.84 \%)$ \\
\hline P.Vivax & $40(30.76 \%)$ & $18(13.84 \%)$ & $58(44.61 \%)$ \\
\hline Mixed & NIL & $2(1.53 \%)$ & $2(1.53 \%)$ \\
\hline \multicolumn{1}{|c|}{ TOTAL } & $\mathbf{8 1 ( 6 2 . 3 0 \% )}$ & $\mathbf{4 9}(\mathbf{3 7 . 6 9 \% )}$ & $\mathbf{1 3 0}$ \\
\hline
\end{tabular}

Table - 2: Sex wise distribution of falciparum and vivax malaria

Maximum numbers (58) of patients were in 20-40 years age group. Other age group patients were 34 in $<20$ years, 33 in 41-60 years age group and 5 were in $>60$ years age group in our study (table 3).

\begin{tabular}{|c|c|c|}
\hline Age group & Number of Patients & \% Age \\
\hline$<20$ yrs & 34 & $26.15 \%$ \\
\hline $21-40$ yrs & 58 & $44.61 \%$ \\
\hline $41-60 y r s$ & 33 & $25.38 \%$ \\
\hline$>60$ yrs & 5 & $3.84 \%$ \\
\hline Total & $\mathbf{1 3 0}$ & $\mathbf{1 0 0} \%$ \\
\hline
\end{tabular}

Table - 3: Distribution of Malaria cases in various age groups

In our study fever was present in $130(100 \%)$ cases, nausea and vomiting 59(45.38\%) cases, headache $42(32.30 \%)$, jaundice in 56(43.07\%), impaired consciousness in 25(19.23\%), oliguria or anuria in $18(13.84 \%)$, bleeding in $9(6.9 \%)$ and convulsion was present in 1 patient. Physical signs on admission were Anemia in 61 (46.92\%), Hepatomegaly in $42(32.30 \%)$, splenomegaly in 68 (52.30\%), hepatosplenomegaly in 36(27.69\%), icterus in 55(42.30\%) and hypotension in 11(8.46\%) were present. During hospitalization total 26 patients developed hypotension, with increase in duration of illness there was increase in hypotension in complicated patients.

On investigation hemoglobin $<7 \mathrm{gm} \%$ was present in 12, hemoglobin $7-10 \mathrm{gm} \%$ in 63 and hemoglobin 10-13 gm\% was present in 55 patients. Total leucocytes $<4000 / \mathrm{mm}$ were in 8,4000 11000 in 109 patients and $>11000$ was present in 13 patients. Serum Bilirubin $>5 \mathrm{gm} \%$ was present in 23 patient, $2-5 \mathrm{gm} \%$ in 33 patients and $<2 \mathrm{gm} \%$ was present in 74 patients. Serum Alanine aminotransferase(ALT) was $>400$ units/L in 5 patients 100-400 units/L in 21 patients, 36-100 units/L in 64 patients and 8-35 units/L in 40 patients. With increase in duration of illness there was increase in incidence of complication (table 4) as icterus was present in 4 patients in 1 day, 12 patients in 1-2 days, 17 patients in 3-5 days and in 22 patients in $>5$ days of illness. Acute renal 
failure in 2 patients in $<1$ day, 5 patients in 1-2 days, 11 patients in 3-5 days and 17 patients in $>5$ days. Cerebral malaria developed in 3 patients in $<1$ day, 5 patients in 1-2 days, 8 patients in $3-5$ days, 9 patients in $>5$ days of illness.

\begin{tabular}{|c|c|c|c|c|}
\hline Duration of illness & \multicolumn{4}{|c|}{ No. of cases of various complication } \\
\cline { 2 - 5 } at hospitalization & Hypotension & Icterus & ARF & Cerebral malaria \\
\hline$<1$ day & $5(19.23 \%)$ & $4(7.27 \%)$ & $2(5.71 \%)$ & $3(12 \%)$ \\
\hline 1-2 days & $6(23.67 \%)$ & $12(21.81 \%)$ & $5(14.28 \%)$ & $5(20 \%)$ \\
\hline $3-5$ days & $7(26.92 \%)$ & $17(30.90 \%)$ & $11(31.42 \%)$ & $8(32 \%)$ \\
\hline > 5 days & $8(30.71 \%)$ & $22(40 \%)$ & $17(48.57 \%)$ & $9(36 \%)$ \\
\hline Table - 4: Table show pattern of complication with duration of \\
illness at the time of hospitalization.(in 59 patient of complicated group)
\end{tabular}

Of the 130 patients neurological involvement was seen in 25 patients of which 8 died, renal involvement was seen in 35 patients of which 3 died, hematological involvement in 83 patients of which 1 died, cardiovascular involvement in 26 patients of which no death was reported, hepatic involvement in 56 patients of which 2, respiratory involvement in 14 patients of which 1 died (table5).

\begin{tabular}{|l|c|c|c|}
\hline System involvement & No. of case & Death & Percentage of death \\
\hline Neurological & 25 & 8 & $53.33 \%$ \\
\hline Renal & 35 & 3 & $20 \%$ \\
\hline Hematological & 83 & 1 & $6.7 \%$ \\
\hline CVS & 26 & - & - \\
\hline Hepatic & 56 & 2 & $13.33 \%$ \\
\hline Respiratory & 14 & 1 & $6.77 \%$ \\
\hline Metabolic & 13 & - & - \\
\hline \multicolumn{4}{|r}{ Table - 5: No. of case of System involvement } \\
\hline
\end{tabular}

This shows that the percentage of mortality in complicated malaria were significantly high more with patient with cerebral malaria (53.33\%).

In present study according of Mohapatra severity score we assessed organ dysfunction from the variables obtained from malaria patients. We obtained 31 cases without any organ dysfunction without any mortality and 36 cases of 1 organ dysfunction without any mortality. There were 23 cases of 2 organ dysfunction with 1 mortality (4.3\%) with score 10.18 cases of 3 organ dysfunction with 1(5.5\%) mortality with score 11 .

8 cases of the 4 organ dysfunction with 2 mortality (25\%) with score 12 and 13.5 cases of the 5 organ dysfunction with 3 mortality (60\%) 1 mortality with malaria severity score 15 and other 2 mortality with malaria severity score $14 \& 16$ respectively. 5 cases of the 6 organ dysfunction with 4 mortality (80\%) 1 mortality with score 16 and second mortality with score 17 and other 2 mortality with score 18. 


\section{ORIGINAL ARTICLE}

4 cases of the 7 organ dysfunction with 4 mortality (100\%) 1 mortality with 19 score and second mortality with malaria severity score 20 and other two mortality with score 21 with p value $0.0001, \chi^{2}=41.5$.

Table 6 shows that total 15 number of patient died and all patient who died had score $>10$ and 9 out of 15 patient who died had score $>16$.

\begin{tabular}{|c|c|c|}
\hline Malaria severity score & No. of case & Death \\
\hline 0 & 31 & 0 \\
\hline 1 & 22 & 0 \\
\hline 2 & 18 & 0 \\
\hline 3 & 12 & 0 \\
\hline 4 & 11 & 0 \\
\hline 5 & 6 & 0 \\
\hline 6 & 4 & 0 \\
\hline 7 & 4 & 0 \\
\hline 8 & 3 & 0 \\
\hline 9 & 1 & 0 \\
\hline 10 & 1 & 1 \\
\hline 11 & 1 & 1 \\
\hline 12 & 2 & 1 \\
\hline 13 & 1 & 1 \\
\hline 14 & 1 & 1 \\
\hline 15 & 1 & 1 \\
\hline 16 & 3 & 2 \\
\hline 17 & 1 & 1 \\
\hline 18 & 2 & 2 \\
\hline 19 & 2 & 1 \\
\hline 20 & 1 & 1 \\
\hline 21 & 2 & 2 \\
\hline Total & 130 & 15 \\
\hline
\end{tabular}

Table - 6: No. of case of malaria severity score

Table 7 shows that increase percentage of mortality with increase in the malaria severity score with significant $P$ value 0.0001 and $X^{2}-50.25$.

\begin{tabular}{|c|c|c|c|}
\hline Malaria severity score & No. of case & Death & Percentage of death \\
\hline $0-7$ & 108 & Nil & - \\
\hline $8-14$ & 10 & 5 & $50 \%$ \\
\hline $15-21$ & 12 & 10 & $83.33 \%$ \\
\hline
\end{tabular}


Table 8 shows that increase percentage of mortality with increase in the number of involvement of organ with 'P' value - 0.0001, $\mathrm{X}^{2}-41.5$.

\begin{tabular}{|c|c|c|c|}
\hline No complication & 31 & 0 & 0 \\
\hline One organ dysfunction & 36 & 0 & 0 \\
\hline Two organ dysfunction & 23 & 1 & $4.3 \%$ \\
\hline Three organ dysfunction & 18 & 1 & $5.5 \%$ \\
\hline Four organ dysfunction & 8 & 2 & $25 \%$ \\
\hline Five organ dysfunction & 5 & 3 & $60 \%$ \\
\hline Six organ dysfunction & 5 & 4 & $80 \%$ \\
\hline Seven organ dysfunction & 4 & 4 & $100 \%$ \\
\hline Total & 130 & 15 & \\
\hline
\end{tabular}

DISCUSSION: Aim of the present study is to assess the importance of a severity score designed by M.K. Mohapatra ${ }^{7}$ for the assessment of disease severity and risk prediction in adult patients of severe malaria.

The present Malaria Severity Score (MSS) ${ }^{7}$ is a physiological scoring system that has been developed to assess the disease severity and to estimate the probability of mortality risk among patients with severe falciparum malaria. The present study also showed that severe malaria is a variable disease causing dysfunction of various organs in different combinations and with variable grades of severity. It can also be applied to estimate the daily risk of mortality and determination of resolution time of Organ Dysfunction.

Of the 7 types of Organ dysfunction neurologic and renal dysfunctions were the most severe with the maximum score of 5 points. Pulmonary, cardiac, and hematologic dysfunctions received 3 points for the most severe level of dysfunction according to table C in M. K. Mohapatra scoring system. Hepatic and metabolic dysfunction received 1 point each, according to M.K. Mohapatra scoring system.

Each Organ dysfunction has been assigned a gradation of severity and allocated a score. Hence, the severity of each OD can be determined quantitatively with the corresponding score and the total score can be obtained by adding the scores of individual OD present in the patient to estimate the risk of mortality.

It can be as low as 1 or as high as 5 with 1 organ dysfunction, and as low as 7 or as high as 21 with all 7 organs in dysfunction. . There are several clinical situations by which a patient might receive high severity score. Either a patient has involvement of several organs with low (level-I) to moderate (level-II) level of dysfunction or few organs with severe level (level-III) of dysfunction. According to table B \& C of malaria severity score of M.K. Mohapatra7 ${ }^{7}$. In any such clinical situation the mortality risk is very high.

This study comprised of 130 patients of malaria admitted in Medical wards from June 2010 to Oct 2011.

Total No. of patients was 130, 70 patients were plasmodium falciparum, 58 patient were plasmodium vivax and 2 patient were mixed malaria.

Malaria severity score is determined for the assessment of the degree of severity, 12 different variables were extracted from the data base and grouped according to system. The variables were analyzed for defining organ dysfunction (OD) and assessment of severity of OD. 
In the present study maximum number of cases was seen in 20-40 yrs. age group. Out of 130 cases, $92(70.71 \%)$ patients were of 20-40 years age group. The increase in man vector contact and immunity may be responsible for this.

In this study major symptoms observed were fever (100\%), nausea and vomiting (45.30\%), headache $(32.30 \%)$, jaundice (43.07\%), cough (16.15\%), pain in abdomen $(21.53 \%)$, impaired consciousness $(19.23 \%)$, and oliguria or anuria (13.84\%).

Gautam A.S. et al (1991) ${ }^{11}$ reported fever in 97\% cases, Gopinathan V.P. et al (1981) ${ }^{12}$ reported fever in $97.8 \%$ and vomiting in $42.2 \%$ cases, Chander et al (1989) ${ }^{13}$ reported fever in $92.7 \%$ and vomiting in $30.9 \%$ cases.

Murthy GL et al (April 2000) ${ }^{14}$ reported fever with Chills and Rigor (98.10\%), altered sensorium (48.10\%), algid malaria (18.35\%), jaundice (27.12\%) cases. Muddaiah Madhu et al $(2006)^{15}$ reported fever in $100 \%$. Nausea and vomiting in $37.36 \%$, headache in $33.6 \%$, jaundice in $15.78 \%$ and altered level of consciousness in $4.21 \%$ cases. Hassan Ali et al (2008) ${ }^{16}$ reported fever in $100 \%$ cases, jaundice in $47.36 \%$, impaired consciousness in $46.05 \%$ cases.

The relative weight of each variable was determined by the coefficients by Mohapatra et al 7 . The strength of the association of each variable with mortality was determined from the least to the most severe level. For example, the coefficient of level of severity for the least severe level of the GCS was 0.50 , and it was 2.58 for the most severe level. The coefficient ranged from 0.22 for the least severe level of Bilirubin to 0.52 for the most severe level of Bilirubin. Therefore, the least severe level of the GCS had the same association with mortality as the most severe level of Bilirubin (table 9).

\begin{tabular}{|c|c|c|c|c|}
\hline Organ system \& variable & 0 & I & II & III \\
\hline \multicolumn{5}{|l|}{ Neurological } \\
\hline Glasgow coma scale & 0.20 & 0.50 & 1.14 & 1.78 \\
\hline \multicolumn{5}{|l|}{ Cardiovascular } \\
\hline Heart rate & 0.24 & 0.55 & 1.24 & \\
\hline Syst. Blood Pressure & 0.21 & 0.57 & 1.12 & \\
\hline \multicolumn{5}{|l|}{ Renal } \\
\hline Urine output & 0.32 & 0.45 & 1.02 & 1.54 \\
\hline Creatinine & 0.31 & 0.47 & 1.08 & 1.57 \\
\hline Urea & 0.44 & 0.80 & 1.13 & 1.7 \\
\hline \multicolumn{5}{|l|}{ Respiratory } \\
\hline Resp.rate & 0.24 & 0.54 & 1.02 & \\
\hline \multicolumn{5}{|l|}{ Hematological } \\
\hline TLC & 0.28 & 0.69 & 1.07 & \\
\hline Platelet & 0.22 & 0.7 & 1.01 & \\
\hline $\mathrm{Hb}$ & 0.2 & & & \\
\hline \multicolumn{5}{|l|}{ Hepatic } \\
\hline Bilirubin & 0.22 & 0.52 & & \\
\hline Metabolic & 0.22 & 0.7 & & \\
\hline
\end{tabular}


In present study jaundice was present in $43.07 \%$ cases. According to malaria severity score serum Bilirubin $>2$ considered as a hepatic dysfunction assigned 1 level of severity and received 1 point. For hepatic dysfunction it is a more severe level of dysfunction but as per Mohapatra it is assigned less score as it has least strength of association with mortality.

Oliguria or Anuria was present in $13.84 \%$ patients. In study according to malaria severity score urine output $0.5-0.75,0.4-0.5,<0.5$ received I, II, III level of severity respectively. The score may be as low as 1 or as high as 5 .

In present study impaired consciousness was present in $18.46 \%$ cases. In present study according to table no. A, B \& $\mathrm{C}$ of the malaria severity score impaired consciousness by GCS from 14$15,10-13,7-9,0-6$ received 0 , I, II, III level of severity respectively. A severity score of $0,1,3$ and 5 was assigned to 0 , I, II, III level of severity respectively. Maximum score is 5 . Hence it is considered most severe form of OD. In present study 17 have 10-13 GCS score received 1 point, 3 patient have 79 GCS score received 2 point, 5 patients 0-6 GCS score assigned III level of severity and received 5 points with maximum severity.

In present study anemia was found in 61 (46.92\%) cases, Hepatomegaly was observed in 42 (32.30\%), splenomegaly in $68(52.30 \%)$ cases and hepatosplenomegaly was found in $26(27.69 \%)$ cases. Anemia results from accelerated RBC removal by spleen, obligatory RBC destruction at parasite schizogony and ineffective erythropoiesis. Enlargement of liver and spleen occur due to congestion as a result of inflammatory response to plasmodia which is more severe in cases of plasmodium falciparum infection.

Murthy GL et al (2000), ${ }^{14}$ Muddaiah Madhu et al (2006) ${ }^{15}$ and Hassan Ali et al (2008) ${ }^{16}$ reported anaemia in $14.27 \%$ cases. Tamal Deb et al (1992) ${ }^{17}$ have observed palpable spleen in $45.6 \%$, Chander et al (1989) ${ }^{13}$ in 40.8\% cases and Gopinathan et al (1982) ${ }^{12}$ in 38.5\% cases which were consistent with study. Incidence of hepatomegaly reported by Tamal Deb et al (1992) ${ }^{17}$ was $25.6 \%$ which was similar to Chander et al (1989) ${ }^{13}$ while Gopinathan V.P. et al (1981) ${ }^{12}$ found this in 15\% of cases. Hazra B.R. et al (1998) ${ }^{18}$ observed splenomegaly in $40 \%$ and hepatomegaly in $80 \%$ cases.

According to malaria severity score, Systolic blood pressure 90-160 mmHg, 70-80 mmHg, 41$69 \mathrm{mmHg}$ assigned 0 , I, II level of severity respectively and received $0,1,3$ points respectively. They are considered as a moderately severe form of the organ dysfunction. In present study, 3 patients have BP 70-80 mmHg assigned level I of severity and received 1 point and 10 patients $<60$ B.P. in assigned II level of severity and received 3 points for maximum severity.

In present study, hypotension was present in 26 out of 130 patient, out of 26 maximum number of hypotensive patient were present with duration of illness $>5$ days. In this point of view if malaria severity score is applied within 48 hours of the illness it is helpful in risk stratification and planning of the treatment to arrest the progression of disease.

High mortality rate of $60 \%$ was observed in hypotension. 9 out of 15 patients with hypotension in the study died. None of the patient died who had hypotension as single complication.

According to M.K. Mohapatra scoring system, Hb 10-13, 7-9.9 and < 7.0 assigned 0, I, II level of severity and received $0,1,3$ points, patient with severe anemia $\mathrm{Hb}<7.0$ assigned level II and received 3 points for maximum severity for hematological dysfunction according to M.K. Mohapatra scoring system. Hence it is considered moderately severe form of organ dysfunction in severity score.

Thrombocytopenia is a common observation in falciparum malaria. The mechanism of thrombocytopenia is poorly understood. The thrombocytopenia is thought to be caused by increased 
splenic sequestration, immune mediated destruction and shortened platelet survival. The degree of thrombocytopenia is associated with severity of falciparum malaria. In most of cases, spontaneous recovery occurs with treatment. In present study, according to Mohapatra scoring system 7 , platelet count 80,000 and $<80,000$ assigned 0 and I level respectively and received 0 and 1 point respectively. It is considered least severe form of the organ dysfunction in M.K. Mohapatra scoring system.

In present study, out of 15 patients who died in the study, 5 patients had severe thrombocytopenia (i.e. platelets $<50,000$ ) and 3 patients had platelets count between $50,000-1$ lacks while 7 patients had normal platelets count. None of the patient who died had thrombocytopenia as a single complication.

In present study 15 patient who died, 12 were having the hyperbilirubinemia associated with the complication likes cerebral malaria, ARF, hypotension. 4 out of 12 patients were present with the SGPT 10 times of the normal, 3 out of 4 patient of plasmodium falciparum and 1 patient of plasmodium vivax who had SGPT 10 times of normal died. Since our study table no. 10 shows elevated liver enzymes (SGPT) we considered that it should also be included as a marker of severity in complicated malaria and should be considered in malaria severity score.

\begin{tabular}{|c|c|c|c|c|c|c|}
\hline \multirow{3}{*}{ SGPT } & \multicolumn{6}{|c|}{ No. of case } \\
\cline { 2 - 7 } & \multicolumn{3}{|c|}{ Uncomplicated } & \multicolumn{3}{c|}{ Complicated } \\
\cline { 2 - 7 } & $\begin{array}{c}\text { P.Falciparum } \\
\mathbf{n = 3 1}\end{array}$ & $\begin{array}{c}\text { P.vivax } \\
\mathbf{n = 4 0}\end{array}$ & $\begin{array}{c}\text { mixed } \\
\text { malaria }\end{array}$ & $\begin{array}{c}\text { P.Falciparum } \\
\mathbf{n = 3 9}\end{array}$ & $\begin{array}{c}\text { P.Vivax } \\
\mathbf{n = 1 8}\end{array}$ & $\begin{array}{c}\text { Mixed } \\
\text { malaria n=2 }\end{array}$ \\
\hline $8-35$ & $15(48.38 \%)$ & $15(37.5 \%)$ & - & $7(17.94 \%)$ & $3(16.6 \%)$ & - \\
\hline $36-100$ & $15(48.38 \%)$ & $25(62.5 \%)$ & - & $15(38.46 \%)$ & $8(44.4 \%)$ & $1(50 \%)$ \\
\hline $100-400$ & $1(3.2 \%)$ & - & - & $13(33.3 \%)$ & $6(33.3 \%)$ & $1(50 \%)$ \\
\hline .400 & - & - & - & $4(10.2 \%)$ & $1(5.5 \%)$ & - \\
\hline
\end{tabular}

Table - 10: Table of Biochemical marker - SGPT

Above table shows that majority of patient who had SGPT 10 time of normal were P.falciparum in complicated group.

In present study, 18 out of 130 patients present with oliguria and anuria. 12 out of 18 had oliguria and 6 out of 18 had anuria. Oliguria or Anuria was present in $13.84 \%$ patients. In study according to malaria severity score urine output $0.5-0.75,0.4-0.5,<0.4$ received I, II, III level of severity respectively. The score may be as low as 1 or as high as 5 .

In present study according to Mohapatra scoring system 7 , blood urea between $10-36 \mathrm{mg} / \mathrm{dl}$, $37-59 \mathrm{mg} / \mathrm{dl}, 60-119 \mathrm{mg} / \mathrm{dl}>120 \mathrm{mg} / \mathrm{dl}$ assigned 0 , I, II, III level of severity and received 0, 1, 3, 5 points respectively.

In our study, 29 patients has blood urea $37-59 \mathrm{mg} / \mathrm{dl}$ assigned I level of severity and received 1 point and 09 patients had blood urea 61-119 assigned II level of severity and received 3 points. 14 had blood urea $>120 \mathrm{mg} / \mathrm{dl}$ assigned III level of severity and received 5 points with maximum level of severity.

According to M.K. Mohapatra malaria severity scoring system 7 , serum creatinine from 0.6-1.2, 1.3-1.9, 2-4.9, > $5 \mathrm{mg} / \mathrm{dl}$ received 0 , I, II, III level of severity and assigned $0,1,3,5$ points. 16 patients had serum creatinine between 1.3-1.9 assigned I level of severity and received 1 point. 12 patients have serum creatinine between $2-4.9 \mathrm{mg} / \mathrm{dl}$ assigned II level of severity received 3 points of severity. 


\section{ORIGINAL ARTICLE}

3 patients have creatinine $>5$ assigned III level of severity and received 5 points of severity with maximum level of severity.

Urine output, blood urea, serum creatinine were grouped together as a renal system. In present study, 12 out of 130 patients who developed ARF had died associated with other complication. Patient who died due to renal involvement had II and III level of severity.

In present study maximum number of patients 18 out of 35 (51.42\%) who developed ARF was present on the third day of hospitalization with $\mathrm{p}$ value 0.009 . So if malaria severity score is applied within first $48 \mathrm{hrs}$. of illness it will be helpful to identify number \& severity of disease \& planning of treatment to arrest progression of disease.

Bajiya et al (1996) ${ }^{19}$ reported high incidence $21.7 \%$, while similar results was observed by Gopinathan et al (1981) ${ }^{12}$, Hazra B.R. et al (1998) ${ }^{18}$ and Prakash J et al (2003) ${ }^{20}$.

Out of 25 cases of cerebral malaria, 12 patients presented with unarousable coma, 6 patients with delirium, 5 with confusion and 2 with psychiatric symptoms. On physical examination, 2 had neck stiffness, 8 had extensor plantar, 2 had papilledema and 6 had retinal hemorrhages, 1 patient presented with generalized tonic clonic seizures along with coma who subsequently died.

In present study impaired consciousness was present in $19.23 \%$ cases. In present study according to the malaria severity score impaired consciousness by GCS from 14-15, 10-13, 7-9, 0-6 received 0 , I, II, III level of severity respectively. A severity score of $0,1,3$ and 5 was assigned to 0 , I, II, III level of severity respectively. Maximum score is 5 . Hence it is considered most severe form of OD. In present study 17 have 10-13 GCS score received 1 point, 3 patient have 7-9 GCS score received 2 point, 5 patients 0-6 GCS score assigned III level of severity and received 5 points with maximum severity for neurological dysfunction according to scoring system.

In present study 8 out of 25 (53.33\%) patients died due to cerebral malaria. Maximum number of patient who developed several malaria were reported on third day of hospitalization and with duration of illness $>5$ day ( $p$ value 0.1 ). So again if malaria score is applied early it will be helpful in risk stratification and further treatment planning.

Dhamija et al (1994) ${ }^{6}$ have reported cerebral malaria in $20 \%$ cases which was consistent with our observations. Murthy GL et al (2000) ${ }^{14}$ reported the cerebral malaria in $45.56 \%$ cases.

Bajiya et al (1996) ${ }^{19}$ reported mortality rate of $33.5 \%$, while Ahmed S.H. et al $(1986)^{21}$ reported mortality in cerebral malaria rate of $23 \%$.

In present study according to malaria severity score, respiratory rate 31-40 and respiratory rate $>41$ assigned level I and II respectively and received 1 and 3 points with maximum level of the severity. It is moderately severe form of the organ dysfunction in present study, 8 patients present with $\mathrm{RR}=31-40$ min assigned level $\mathrm{I}$ and received 1 point and 3 patients present with $\mathrm{RR}>41$ assigned level II received 3 points. 1 patient was died due to malarial ARDS with maximum 3 points.

According to malaria severity score, blood sugar $60-110 \mathrm{mg} / \mathrm{dl}$ assigned level 0 and blood sugar $<60 \mathrm{mg} / \mathrm{dl}$ assigned level I received 1 point. Sodium $<129$, and potassium $<3.5$ assigned level I and received 1 point for maximum severity. It is considered as least severe form of the organ dysfunction. In present study, 12 patients had blood sugar $<60 \mathrm{mg} / \mathrm{dl}$ received 1 point, 8 patients have hyponatremia and 5 patients have hypokalemia. None of the patient who died had metabolic dysfunction as a single complication.

In malaria severity score of Mohapatra, all 12 variables were analyzed to identify cut off points that define normal and abnormal values. Further from the abnormal values, different level of 
severity for each variable was determined. When the values were within the normal range it was considered as level-0 severity. 3 levels of increasing severity i.e. Level-I, II, and III were identified. Variable related to one system were grouped together to define that particular system.

A severity score of $0,1,3$, and 5 was assigned to 0 , I, II, and III level of severity respectively. The score may be as low as 1 or as high as 5 for neurologic and renal involvement. For cardiovascular, respiratory, and hematological system the lowest and the highest score was 1 and 3. For hepatic and metabolic involvement only 1 score was assigned.

Organ dysfunction with only one level (I), two levels (I and II), and three levels (I to III) of severity were considered as least, moderate, and most severe dysfunction.

The points for each organ dysfunction are summed to calculate the total malaria severity score (MSS). MSS of 0 indicates no organ dysfunction, whereas MSS of 1 is the lowest level of severity possible for one single organ dysfunction. The maximum score with 21 points can be found when a patient has all 7 organs dysfunction with their highest level of severity.

Risk prediction according to severity score for each level of severity a severity score has been assigned and subsequently for each value of severity score the probability of hospital mortality has been calculated. The patients are grouped as i) Low risk when the score is 7 or less (with low mortality risk) ii) Intermediate risk when the score is within 8 to 14 with a risk of mortality 50\%, and iii) High risk when the severity score is $15-21$ with mortality risk of $88.33 \%$ or above $(\mathrm{p}<0.001)$.

In present study according of Mohapatra severity score ${ }^{7}$ we assessed organ dysfunction from the variables obtained from malaria patients. We obtained 31 cases without any organ dysfunction without any mortality and 36 cases of 1 organ dysfunction without any mortality. There were 23 cases of 2 organ dysfunction with 1 mortality (4.3\%) with score 10.18 cases of 3 organ dysfunction with $1(5.5 \%)$ mortality with score 11 .

8 cases of the 4 organ dysfunction with 2 mortality (25\%) with score 12 and 13.5 cases of the 5 organ dysfunction with 3 mortality (60\%) 1 mortality with malaria severity score 15 and other 2 mortality with malaria severity score $14 \& 16$ respectively. 5 cases of the 6 organ dysfunction with 4 mortality (80\%) 1 mortality with score 16 and second mortality with score 17 and other 2 mortality with score 18.

4 cases of the 7 organ dysfunction with 4 mortality (100\%) 1 mortality with 19 score and second mortality with malaria severity score 20 and other two mortality with score 21 with p value $0.0001, \chi^{2}=41.5$.

As per table all the complication were reported within 3-5 days of illness or 2nd and 3rd day of hospitalization. In this point of view if malaria severity score is applied within 48 hours of illness it will be helpful for risk stratification and planning of the treatment to arrest the progression of disease.

In present study 15 out of 130 patients died, 4 were plasmodium vivax and 11 were plasmodium falciparum.

Of the 5 species of the plasmodium falciparum causing most serious form of the disease in patient with this parasite may become serious and fatal. Plasmodium falciparum schizont release an average of the 16 merozoites and each infect and other RBC causing infective biomass to expand a exponentially. Plasmodium falciparum infects erythrocytes of any age with the potential of development of high-grade parasitemia. In present study we reported 11 deaths due to the plasmodium falciparum b/o high grade parasitemia. 
Rest 4 deaths were due to plasmodium vivax. These patients may have died due to the isolated plasmodium vivax malaria or may be due to the mixed malaria in which plasmodium falciparum was not detected in peripheral smear. 2 out of 4 patient had significant septicemia so may be the possibility of severe sepsis coincidently present with plasmodium vivax malaria. Due to the changing trend of the plasmodium vivax presenting in the severe form, it should not be taken lightly.

\section{CONCLUSION:}

- Most common clinical presentations of Malaria were fever, nausea and vomiting, headache.

- Increase in the duration of illness is related to increase in the severity of disease

- Incidence of mortality significantly increases with increase in the malaria severity score in malaria patient.

- Incidence of mortality significantly increases with increase in the number of organ involvement.

- In case of malaria, just detection of P. falciparum parasite in peripheral smear can make the diagnosis but assessment of malaria severity score help in assessing severity of organ dysfunction in required for risk stratification, prognosis and planning of the treatment to arrest the progression of disease as it is cheap \& effective it should be done in all malaria patients.

- It can be used for daily risk estimate and to determine the resolution time of each complication.

- In our study elevated liver enzymes (SGPT) were significantly associated with increased mortality. We suggest it should also be included as a marker of severity in complicated malaria and should be considered in malaria severity score.

\section{BIBLIOGRAPHY:}

1. World Health organisation, World Malaria Situation in 1992, weekly epid report 1994; 69, 30932.

2. K. Park, Text book of Preventive \& Social Medicine 20th Edition; 223.

3. Kochar DK, Kochar SK, Agrawal RP, Sabir M, Nayak KC, Agrawal TD, et al. The changing spectrum of severe P. falciparum malaria: a clinical study from Bikaner (North West India). J Vector Borne Dis 2006; 43:104-8.

4. Mohapatra MK. The Natural history of complicated P. falciparum malaria; a prospective study. J Assoc. Physicians India 2006; 54:848-53.

5. Gupta A, Malhotra hS, Mittal PC, Mahajan RC, Sharma BK, Deondnar SD. Clinical pattern of complicated malaria in North West India. JAPI 1987; 35:15.

6. Dhamija RM. Cerebral malaria: Indian scene, Medicine Update, Vol. 4 (APICON-94), Ed. Walial PK PIII: 11-19.

7. Mohapatra MK, Das SP. The malaria severity score: a method for severity assessment and risk prediction of hospital mortality for falciparum malaria in adults. J Assoc Physicians India. 2009 Feb;57:119-26

8. Mishra SK, Panigrahi P, Mishra R, Mohanty S. Prediction of outcome in adults with severe falciparum malaria: a new scoring system. Malaria Journal 2007; 6:24-28. 
9. Knaus WA, Wagner DP, Draper EA, Zimmerman JE, et al. The APACHEIII prognostic system-Risk prediction of hospital mortality for critically ill hospitalized adults. Chest 1991; 100:1619-36.

10. LeGall J, Klar J, Lemeshow S, Saulnier F, et al. The logistic organ dysfunction system: a new way to assess organ dysfunction in the intensive care unit. J Am Med Asso 1996; 276:802-10.

11. Gautam AS, Sharma RC, Sharma VP, Sharma GK. Importance of clinical diagnosis of malaria in National Malaria Control Programme. Indian J of Malariology 1991; 183-187.

12. Gopinathan VP, Ratla PK, Bhopte AG. Falciparum malaria in North Eastern Sector JAPI Ind. Vol. 29 Dec. 1981.

13. Chander V, Mehtra SR, Sharma PD, Sarkar PK, Sharma BR. Falciparum malaria. Indian J Pediatr. 1989; 56:365-369.

14. Murthy GL, Sahay RK, Srinivasan VR, Upadhaya AC, Shantaram V, Gayatri K. Clinical profile of falciparum malaria in a tertiary care hospital. J Indian Med Assoc. 2000 Apr; 98(8):160-2, 169.

15. Muddaiah Madhu \& Prakash PS. A study of clinical profile of malaria in a tertiary referral centre in South Canara. J Vect Bome Dis 43; March 2006; 29-33.

16. Hassan Ali, Tasnim Ahsan; Tariq Mohmood, Syed Feroze Bakht, Mohammd Umer Farooq and Niaz Ahmed. Parasite density and spectrum of clinical illness in falciparum malaria. Journal of the College of Physicians and Surgeons, Pakistan 2008; Vol. 18(6); 362-368.

17. Tamal D, Mohanti RK, Ravi K, Bhagat BM. Atypical presentations of falciparum malaria. JAPI 1992 Jan; 40(6): 381-4.

18. Hazra BR, Chowdhury RS, SahaSk, Ghosh MB, Mazumder AK. Changing scenario of malaria: a study at Calcutta. Indian J Malariol. 1998 Jun; 35(2):111-6.

19. Bajiya HN, Kochar DK. Incidence of outcome of neurological sequelae in survivors of cerebral malaria. JAPI 1996; Vol. 44, No. 10; 679-681.

20. Prakash J, Singh AK, Kumar NS, Saxena RK. Acute renal failure in plasmodium vivax malaria. JAPI March 2003; 51:265-7.

21. Ahmed SH, Moonis R. Cerebral malaria in children. Indian J. Pediatr. 53:409-13.

\section{AUTHORS:}

1. Aharwar S.

2. Kansal A.

3. Jatav O. P.

4. Malviya K.

5. Trikha S.

\section{PARTICULARS OF CONTRIBUTORS:}

1. Assistant Professor, Department of Medicine, Gajra Raja Medical College, Gwalior (M.P.)

2. Associate Professor, Department of Medicine, Gajra Raja Medical College, Gwalior (M.P.)

3. Professor and Head, Department of Medicine, Gajra Raja Medical College, Gwalior (M.P.)

4. Associate Professor, Department of Medicine, Gajra Raja Medical College, Gwalior (M.P.)
5. Resident, Department of Medicine, Gajra Raja Medical College, Gwalior (M.P.)

\section{NAME ADDRESS EMAIL ID OF THE} CORRESPONDING AUTHOR:

Dr. Sandeep Aharwar,

M-241, Madhav Nagar,

Opposite A. G. Office,

Lashkar, Gwalior (M.P.)

E-mail: sandeepaharwar31@rediffmail.com

Date of Submission: 20/01/2014.

Date of Peer Review: 21/01/2014.

Date of Acceptance: 31/01/2014.

Date of Publishing: 17/02/2014. 